\title{
THE CORRELATION BETWEEN DISPERSION MEASURE AND X-RAY COLUMN DENSITY FROM RADIO PULSARS
}

\author{
C. $\mathrm{HE}^{1,2}$, C.-Y. $\mathrm{NG}^{1,3}$, AND V. M. KASPI ${ }^{1}$ \\ ${ }^{1}$ Department of Physics, McGill University, Montreal, QC H3A 2T8, Canada; ncy@ bohr.physics.hku.hk \\ 2 Department of Physics, University of Chicago, Chicago, IL 60637, USA \\ ${ }^{3}$ Department of Physics, The University of Hong Kong, Pokfulam Road, Hong Kong \\ Received 2012 December 20; accepted 2013 March 18; published 2013 April 15
}

\begin{abstract}
Pulsars are remarkable objects that emit across the entire electromagnetic spectrum, providing a powerful probe of the interstellar medium. In this study, we investigate the relation between dispersion measure (DM) and $\mathrm{X}$-ray absorption column density $N_{\mathrm{H}}$ using 68 radio pulsars detected at X-ray energies with the Chandra $X$-Ray Observatory or XMM-Newton. We find a best-fit empirical linear relation of $N_{\mathrm{H}}\left(10^{20} \mathrm{~cm}^{-2}\right)=$ $0.30_{-0.09}^{+0.13} \mathrm{DM}\left(\mathrm{pc} \mathrm{cm}^{-3}\right)$, which corresponds to an average ionization of $10_{-3}^{+4} \%$, confirming the ratio of one free electron per 10 neutral hydrogen atoms commonly assumed in the literature. We also compare different $N_{\mathrm{H}}$ estimates and note that some $N_{\mathrm{H}}$ values obtained from X-ray observations are higher than the total Galactic $\mathrm{H}$ I column density along the same line of sight, while the optical extinction generally gives the best $N_{\mathrm{H}}$ predictions.
\end{abstract}

Key words: dust, extinction - ISM: general - pulsars: general - X-rays: ISM

Online-only material: color figures

\section{INTRODUCTION}

The broadband emission of pulsars from radio frequencies to $\gamma$-rays can be used to probe the physical conditions of the interstellar medium (ISM). Specifically, their radio pulsations allow accurate measurements of the free electron column density and their X-ray extinction traces the interstellar gas along the line of sight. Radio waves traveling in the ISM are dispersed by free electrons such that signals at lower frequencies propagate at a lower speed and hence arrive on Earth later than those at higher frequencies. The time delay $(\Delta t)$ between two observing frequencies $\left(v_{1}, v_{2}\right)$ depends on the dispersion measure (DM), which is the integrated free electron number density $n_{e}$ from Earth to the source at distance $d$ :

$$
\mathrm{DM}=\int_{0}^{d} n_{e} d l=\frac{2 \pi m_{e} c}{e^{2}}\left(\frac{1}{v_{1}^{2}}-\frac{1}{v_{2}^{2}}\right)^{-1} \Delta t
$$

where $m_{e}$ and $e$ are electron mass and charge, respectively, and $c$ is the speed of light. Most free electrons in our Galaxy are found in the hot phase of the ISM, including H II regions ionized by UV radiation from hot $\mathrm{O}$ or $\mathrm{B}$ type stars and the shock-heated interior of supernova remnants (SNRs). These sources can contribute significant DM up to a few hundred parsecs per cubic centimeter. At X-ray energies, photons are absorbed mostly by heavy elements in the interstellar gas due to the photoelectric effect. This has a strong energy dependence and is most prominent in the soft X-ray band. As a result, it modifies the observed low-energy portion of the X-ray spectrum and has to be accounted for in spectral modeling. The amount of extinction, which is expressed in terms of the equivalent atomic hydrogen column density $N_{\mathrm{H}}$, is sensitive to gas and molecular clouds, which traces the warm and cold phases of the ISM (see Wilms et al. 2000).

One natural question to ask is whether there is any correlation between DM and $N_{\mathrm{H}}$ in our Galaxy. Such a correlation can reflect the physical connection between different phases of the ISM. Also, it can provide a useful tool to estimate one quantity from the other, help plan new observations, and determine $\mathrm{X}$-ray luminosity upper limits in cases of non-detection. In the literature, an average ionization fraction of $10 \%$ in the ISM, i.e., one free electron per 10 equivalent hydrogen atoms, has been commonly assumed in order to infer $N_{\mathrm{H}}$ from DM (e.g., Seward \& Wang 1988; Kargaltsev et al. 2007; Gil et al. 2008; Camilo et al. 2012), but the justification for this choice has been unclear. $\mathrm{X}$-ray-emitting radio pulsars offer a powerful diagnostic tool for a quantitative study of the correlation. Because they are model independent and relatively straightforward to measure from radio timing, DM values are well determined, typically better than a fractional uncertainty of $10^{-3}$. However, what has made the determination of any $\mathrm{DM}-N_{\mathrm{H}}$ correlation difficult in the past is the lack of high-quality X-ray data for $N_{\mathrm{H}}$ measurements. In particular, previous generations of X-ray telescopes had poor angular resolution that precluded discerning the pulsar emission from that of the surrounding SNRs and pulsar wind nebulae (PWNe). Thanks to new X-ray missions such as the Chandra $X$-Ray Observatory and XMM-Newton, precise measurements of $N_{\mathrm{H}}$ have been obtained for many pulsars in recent years, allowing a statistical study of $N_{\mathrm{H}}$ values for the first time.

In this paper, we compile a list of DM and $N_{\mathrm{H}}$ values for $68 \mathrm{X}$-ray-emitting radio pulsars using the latest Chandra and $X M M$-Newton measurements reported in the literature. We found a clear correlation between these two column densities and obtained a best-fit empirical relation of $10_{-3}^{+4} \%$ ionization. In Section 2, we describe our sample selection criteria. The statistical analysis and results are presented in Section 3, and we discuss the implications of our results in Section 4.

\section{SAMPLE SELECTION}

We started with a list of X-ray-detected radio pulsars from Possenti et al. (2002), Becker \& Aschenbach (2002), Pavlov et al. (2007), Kargaltsev \& Pavlov (2008), and Kargaltsev \& Pavlov (2010), then expanded the sample through careful literature searches for updated observational results and recent discoveries. The latter include three magnetars that show radio emission (Camilo et al. 2006, 2007; Levin et al. 2010) and over a 
dozen new pulsars identified in $\gamma$-rays with the Fermi Gammaray Space Telescope and subsequently detected in follow-up radio and X-ray observations (see Marelli et al. 2011). Finally to complete the list, we went through the Chandra and XMMNewton data archive to search for pulsar observations, and looked up relevant publications based on these data.

The pulsar DMs are adopted from the ATNF Pulsar Catalog 4 (Manchester et al. 2005). They are all very well measured with negligible uncertainties compared to those for $N_{\mathrm{H}}$. On the other hand, it is much more difficult to determine $N_{\mathrm{H}}$, because this requires a strong $\mathrm{X}$-ray source and good knowledge of the intrinsic emission spectrum. The X-ray emission of pulsars is not fully understood; commonly used models include a blackbody (BB) and a neutron-star hydrogen atmosphere (NSA) for the thermal emission, and a power law (PL) for the non-thermal emission. More complicated models consisting of thermal and non-thermal components are sometimes used. To minimize any bias, we selected the $N_{\mathrm{H}}$ values for our sample according to the following criteria.

1. We restricted our choices to those in the latest studies using the Chandra and XMM-Newton observations, since the good angular resolution and sensitivity of these telescopes offer high-quality spectra with minimal background contamination. Any joint fits with other X-ray telescopes are not considered, in order to avoid cross-calibration uncertainties.

2. We adopted only $N_{\mathrm{H}}$ values from actual X-ray spectral fits in which the $N_{\mathrm{H}}$ is allowed to vary freely, and ignored any $N_{\mathrm{H}}$ inferred from DM, optical extinction $\left(A_{\mathrm{V}}\right)$, or total Galactic H i column density.

3. $N_{\mathrm{H}}$ from the best-fit spectral model is always preferred, unless there are physical arguments favoring another model. If different emission models give the same goodness of fit and the authors do not indicate a clear preference, we choose the simpler one. For example, we prefer a BB model over an NSA model, since the latter requires more assumptions, including the atmosphere composition, surface magnetic field, and gravity.

4. For pulsars associated with bright PWNe, the nebular $N_{\mathrm{H}}$ values are adopted if they are better constrained than those of the pulsars, because the simple PL spectra of PWNe can reduce systematic uncertainties in spectral modeling. $N_{\mathrm{H}}$ from SNRs are used in a few cases when the pulsars and PWNe are too faint for useful $N_{\mathrm{H}}$ measurements.

Our final sample contains 68 pulsars. One of them (PSR B0540-69) is extragalactic and only two (PSRs J1740-5340 and B1821-24) are in globular clusters; cluster pulsars are generally too faint for precise $N_{\mathrm{H}}$ measurements. The pulsar DM and $N_{\mathrm{H}}$ values are listed in Table 1 and plotted in Figure 1. The reported statistical uncertainties and upper limits for $N_{\mathrm{H}}$ are at $90 \%$ confidence level, i.e., $1.6 \sigma$. We list in the table the X-ray spectral models used to obtain $N_{\mathrm{H}}$. The choice of spectral model is clear in all cases except PSRs J1622-4950 and B1757-24, for which both thermal and non-thermal fits are acceptable. Nonetheless, $N_{\mathrm{H}}$ from different fits only varies by a factor of two for J1622-4950 and does not change for B1757-24. Therefore, we conclude that systematic bias induced by spectral models is minimal.

Table 1 also shows the pulsar Galactic coordinates $(l, b)$ and distances, and this information was used to calculate the vertical height $(z)$ from the Galactic plane. The coordinates are

\footnotetext{
4 http://www.atnf.csiro.au/research/pulsar/psrcat/
}
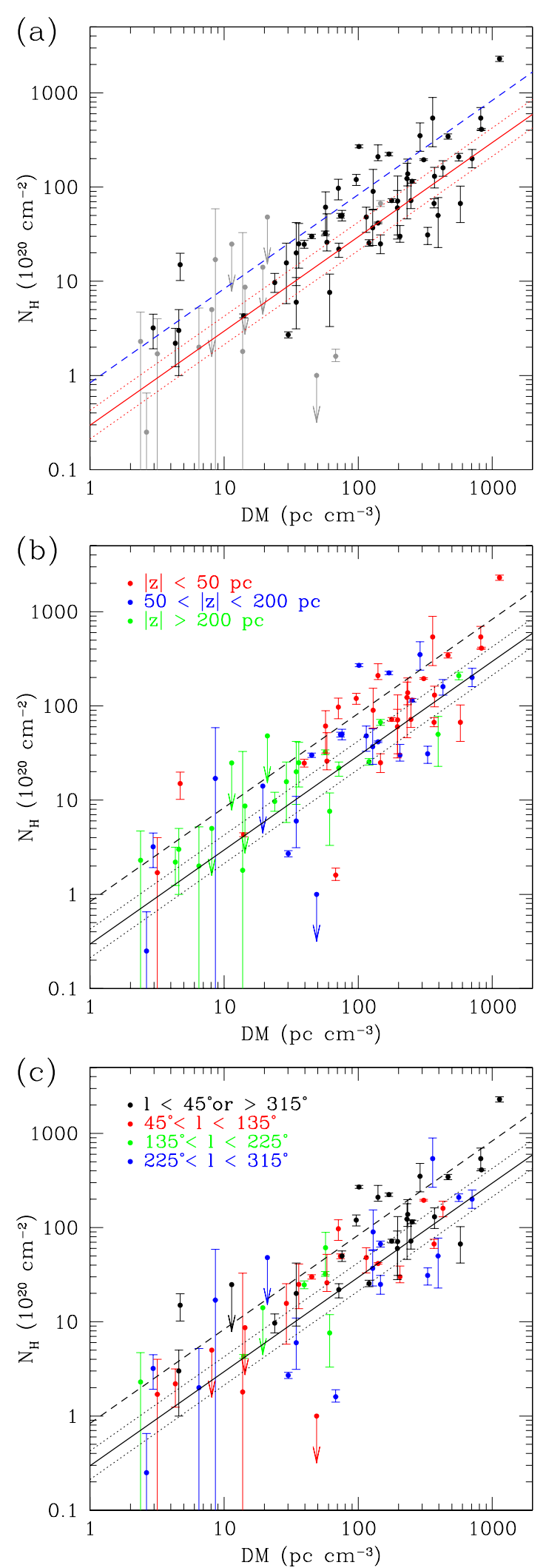

Figure 1. $N_{\mathrm{H}}$ vs. DM for 68 pulsars. (a) Data points in gray color, including the Vela pulsar, PSR B0540-69, and $N_{\mathrm{H}}$ measurements with fractional uncertainties larger than $80 \%$ or only upper limits are not used in the fit. The red solid and dotted lines show the best linear fit with the $90 \%$ confidence interval, and the blue dashed line is the same fit ignoring measurement uncertainties. These correspond to $10_{-3}^{+4} \%$ and $4 \%$ ionization, respectively. Uncertainties in DM are negligible. The same plot is shown in (b) and (c) with different color schemes, indicating the pulsar vertical distance from the Galactic plane and their Galactic longitudes, respectively.

(A color version of this figure is available in the online journal.) 
Table 1

DM, $N_{\mathrm{H}}$, and Distances for the 68 Pulsars Used in This Study

\begin{tabular}{|c|c|c|c|c|c|c|c|c|}
\hline PSR & $\begin{array}{c}\mathrm{DM} \\
\left(\mathrm{pc} \mathrm{cm}{ }^{-3}\right)\end{array}$ & $\begin{array}{c}N_{\mathrm{H}} \\
\left(10^{20} \mathrm{~cm}^{-2}\right)\end{array}$ & $\begin{array}{l}\text { Distance }^{\mathrm{a}} \\
\quad(\mathrm{kpc})\end{array}$ & $\begin{array}{c}l \\
(\mathrm{deg})\end{array}$ & $\begin{array}{c}b \\
(\mathrm{deg})\end{array}$ & $\begin{array}{c}z^{\mathrm{b}} \\
(\mathrm{pc})\end{array}$ & Model $^{\mathrm{c}}$ & Ref. \\
\hline $\mathrm{J} 0030+0451$ & $4.333 \pm 0.001$ & $2.2 \pm 1.0$ & $0.28_{-0.06}^{+0.10 \mathrm{p}}$ & 113.1 & -57.6 & -236 & $\mathrm{NSA} \times 3$ & 1 \\
\hline J0108-1431 & $2.4 \pm 0.2$ & $2 \pm 2$ & $0.21_{-0.05}^{+0.09} p^{2}$ & 140.9 & -76.8 & -205 & BB & 2 \\
\hline B0136+57 & $73.779 \pm 0.006$ & $50 \pm 3$ & $2.6_{-0.2}^{+0.3} \mathrm{p}$ & 129.2 & -4.0 & -183 & PL & 3 \\
\hline $\mathrm{J} 0205+6449$ & $140.7 \pm 0.3$ & $41.6_{-0.7}^{+0.8}$ & $3.2^{\circ}$ & 130.7 & +3.1 & +172 & $\mathrm{PL}+\mathrm{RS}$ (SNR) & 4,5 \\
\hline $\mathrm{J} 0218+4232$ & $61.252 \pm 0.005$ & $8 \pm 4$ & $2.67^{\mathrm{d}}$ & 139.5 & -17.5 & -804 & PL & 6 \\
\hline В0355+54 & $57.1420 \pm 0.0003$ & $60 \pm 30$ & $1.0_{-0.1}^{+0.2 \mathrm{p}}$ & 148.2 & +0.8 & +14 & PL (PWN) & 7 \\
\hline J0437-4715 & $2.64476 \pm 0.00007$ & $0.25_{-0.24}^{+0.40}$ & $0.156 \pm 0.001^{\mathrm{p}}$ & 253.4 & -42.0 & -104 & $\mathrm{PL}+\mathrm{NSA} \times 2$ & 8 \\
\hline B0531+21 (Crab) & $56.791 \pm 0.001$ & $32 \pm 2$ & $2.00^{\circ}$ & 184.6 & -5.8 & -202 & PL & 9,10 \\
\hline J0538+2817 & $39.570 \pm 0.001$ & $25 \pm 2$ & $1.3 \pm 0.2^{\mathrm{p}}$ & 179.7 & -1.7 & -38 & $\mathrm{BB}$ & 11 \\
\hline B0540-69 & $146.6 \pm 0.2$ & $67 \pm 5$ & $50^{\circ}$ & 279.7 & -31.5 & -26137 & PL (SNR) & 12,13 \\
\hline B0628-28 & $34.468 \pm 0.017$ & $6_{-3}^{+5}$ & $0.32_{-0.04}^{+0.05 p}$ & 237.0 & -16.8 & -92.3 & PL & 14 \\
\hline B0656+14 & $13.977 \pm 0.013$ & $4.3 \pm 0.2$ & $0.28 \pm 0.03^{\mathrm{p}}$ & 201.1 & +8.3 & +40 & $\mathrm{PL}+\mathrm{BB} \times 2$ & 15 \\
\hline J0737-3039 & $48.920 \pm 0.005$ & $<1$ & $1.1_{-0.1}^{+0.2 \mathrm{p}}$ & 245.2 & -4.5 & -86 & $\mathrm{BB} \times 2$ & 16 \\
\hline B0823+26 & $19.454 \pm 0.004$ & $<14$ & $0.32_{-0.05}^{+0.08 p}$ & 197.0 & +31.7 & +168 & PL & 17 \\
\hline B0833-45 (Vela) & $67.99 \pm 0.01$ & $1.6_{-0.2}^{+0.3}$ & $0.28 \pm 0.02^{\mathrm{p}}$ & 263.6 & -2.8 & -14 & PL $(\mathrm{PWN})$ & 18 \\
\hline В0950+08 & $2.958 \pm 0.003$ & $3.2 \pm 1.3$ & $0.261 \pm 0.005^{\mathrm{p}}$ & 228.9 & +43.7 & +180 & PL+BB & 19 \\
\hline J1016-5857 & $394.2 \pm 0.2$ & $50 \pm 30$ & $8.00^{\mathrm{d}}$ & 284.1 & -1.9 & -263 & PL & 20 \\
\hline $\mathrm{J} 1023+0038$ & $14.325 \pm 0.010$ & $<9$ & $1.37 \pm 0.04^{\mathrm{p}}$ & 243.5 & +45.8 & +980 & $\mathrm{PL}+\mathrm{NSA}$ & 21 \\
\hline J1024-0719 & $6.48520 \pm 0.00008$ & $2_{-2}^{+3}$ & $0.49_{-0.08}^{+0.12 p}$ & 251.7 & +40.5 & +318 & BB & 22 \\
\hline B1046-58 & $129.1 \pm 0.2$ & $90_{-30}^{+60}$ & $2.9_{-0.7}^{+1.2 \mathrm{~h}}$ & 287.4 & +0.6 & +29 & PL & 23 \\
\hline B $1055-52$ & $30.1 \pm 0.5$ & $2.7 \pm 0.2$ & $0.72^{\mathrm{d}}$ & 286.0 & +6.7 & +84 & $\mathrm{PL}+\mathrm{BB} \times 2$ & 15 \\
\hline J1119-6127 & $707.4 \pm 1.3$ & $200_{-40}^{+50}$ & $8.4 \pm 0.4^{\circ}$ & 292.2 & -0.5 & -78.7 & PL+BB & 24,25 \\
\hline J1124-5916 & $330 \pm 2$ & $31 \pm 6$ & $5_{-2}^{+3 h}$ & 292.0 & +1.8 & +153 & PL & 26 \\
\hline J1231-1411 & $8.090 \pm 0.001$ & $<5$ & $0.44^{\mathrm{d}}$ & 295.5 & +48.4 & +329 & $\mathrm{NSA}+\mathrm{PL}$ & 27 \\
\hline B1259-63 & $146.72 \pm 0.03$ & $25_{-5}^{+6}$ & $2.3 \pm 0.4^{\circ}$ & 304.2 & -1.0 & -40 & PL & 28,29 \\
\hline J1357-6429 & $128.5 \pm 0.7$ & $37_{-13}^{+20}$ & $2.50^{\mathrm{d}}$ & 309.9 & -2.5 & -110 & PL (PWN) & 30 \\
\hline J1400-6325 & $563 \pm 4$ & $209 \pm 20$ & $11.27^{\mathrm{d}}$ & 310.6 & -1.6 & -313 & PL (PWN) & 31 \\
\hline J1420-6048 & $358.8 \pm 0.2$ & $540_{-270}^{+350}$ & $5.61^{\mathrm{d}}$ & 313.5 & +0.2 & +22 & PL (PWN) & 32 \\
\hline B1451-68 & $8.6 \pm 0.2$ & $17_{-17}^{+40}$ & $0.43_{-0.05}^{+0.06} \mathrm{p}$ & 313.9 & -8.5 & -64 & PL+BB & 33 \\
\hline $\mathrm{J} 1509-5850$ & $140.6 \pm 0.8$ & $210_{-20}^{+70}$ & $2.62^{\mathrm{d}}$ & 320.0 & -0.6 & -28 & PL (PWN) & 34 \\
\hline B1509-58 & $252.5 \pm 0.3$ & $115 \pm 5^{\mathrm{d}}$ & $4.4_{-0.8}^{+1.3 \mathrm{~h}}$ & 320.3 & -1.2 & -89 & PL (PWN) & 35 \\
\hline J1550-5418 & $830 \pm 50$ & $410 \pm 10$ & $9.55^{\mathrm{d}}$ & 327.2 & -0.1 & -22 & PL+BB & 36 \\
\hline J1614-2230 & $34.4865 \pm 0.0001$ & $20_{-11}^{+22}$ & $1.27^{\mathrm{d}}$ & 352.6 & +20.2 & +438 & $\mathrm{BB} \times 2$ & 37 \\
\hline J1617-5055 & $467 \pm 5$ & $345 \pm 20$ & $6.82^{\mathrm{d}}$ & 332.5 & -0.3 & -33 & PL & 38 \\
\hline $\mathrm{J} 1622-4950$ & $820 \pm 30$ & $540_{-140}^{+160}$ & $8.73^{\mathrm{d}}$ & 333.8 & -0.1 & -16 & $\mathrm{BB}$ & 39 \\
\hline B1706-44 & $75.69 \pm 0.05$ & $50 \pm 6$ & $2.6_{-0.6}^{+0.5 \mathrm{~h}}$ & 343.1 & -2.7 & -122 & PL (PWN) & 40 \\
\hline J1718-3718 & $371.1 \pm 1.7$ & $130 \pm 30$ & $6.6^{\mathrm{d}}$ & 349.8 & +0.2 & +25 & BB & 41 \\
\hline J1718-3825 & $247.4 \pm 0.3$ & $72_{-13}^{+50}$ & $3.6^{\mathrm{d}}$ & 349.0 & -0.4 & -27 & PL (PWN) & 42 \\
\hline J1734-3333 & $578 \pm 9$ & $70_{-30}^{+40}$ & $6.46^{\mathrm{d}}$ & 354.8 & -0.4 & -49 & BB & 43 \\
\hline J1740-5340 & $71.8 \pm 0.2$ & $22 \pm 4$ & $2.7 \pm 0.2^{\circ}$ & 338.2 & -12.0 & -560 & PL & 44,45 \\
\hline $\mathrm{J} 1740+1000$ & $23.85 \pm 0.05$ & $10 \pm 2$ & $1.24^{\mathrm{d}}$ & 34.0 & +20.3 & +430 & $\mathrm{PL}+\mathrm{BB}$ & 46 \\
\hline J1741-2054 & $4.7 \pm 0.1$ & $15 \pm 5$ & $0.38^{\mathrm{d}}$ & 6.4 & +4.9 & +33 & PL $(\mathrm{PWN})$ & 47 \\
\hline J1747-2809 & $1133 \pm 3$ & $2300 \pm 150$ & $13.31^{\mathrm{d}}$ & 0.9 & +0.1 & +18 & PL $(\mathrm{PWN})$ & 48 \\
\hline J1747-2958 & $101.5 \pm 1.6$ & $270 \pm 10$ & $5^{\circ}$ & 359.3 & -0.8 & -73 & PL (PWN) & 49,50 \\
\hline B1757-24 & $289 \pm 10$ & $350_{-110}^{+130}$ & $5.22^{\mathrm{d}}$ & 5.3 & -0.9 & -80 & PL & 51 \\
\hline B $1800-21$ & $233.99 \pm 0.05$ & $138_{-35}^{+60}$ & $3.88^{\mathrm{d}}$ & 8.4 & +0.2 & +10 & PL (PWN) & 52 \\
\hline J1809-1917 & $197.1 \pm 0.4$ & $71_{-40}^{+60}$ & $3.55^{\mathrm{d}}$ & 11.1 & +0.1 & +5 & PL & 53 \\
\hline J1809-1943 & $178 \pm 5$ & $72 \pm 3$ & $3.6 \pm 0.5^{\mathrm{h}}$ & 10.7 & -0.2 & -10 & $\mathrm{BB} \times 3$ & 54 \\
\hline $\mathrm{J} 1819-1458$ & $196.0 \pm 0.4$ & $60 \pm 30$ & $3.55^{\mathrm{d}}$ & 16.0 & +0.1 & +5 & BB & 55 \\
\hline B1821-24 & $120.502 \pm 0.002$ & $26 \pm 2$ & $5.5 \pm 0.3^{\circ}$ & 7.8 & -5.6 & -535 & PL & 56,57 \\
\hline B1823-13 & $231 \pm 1$ & $120_{-80}^{+60}$ & $3.93^{\mathrm{d}}$ & 18.0 & -0.7 & -47 & PL (PWN) & 58 \\
\hline J1833-1034 & $169.5 \pm 0.1$ & $224_{-10}^{+9}$ & $4.5 \pm 0.5^{\mathrm{h}}$ & 21.5 & -0.9 & -70 & PL & 59 \\
\hline B1853+01 & $96.74 \pm 0.12$ & $120 \pm 16$ & $3^{\circ}$ & 34.6 & -0.5 & -26 & $\mathrm{VNEI} \times 2(\mathrm{SNR})$ & 60,61 \\
\hline $\mathrm{J} 1930+1852$ & $308 \pm 4$ & $195 \pm 4$ & $7_{-2}^{+3 \mathrm{~h}}$ & 54.1 & +0.3 & +32 & PL (PWN) & 62 \\
\hline
\end{tabular}


Table 1

(Continued)

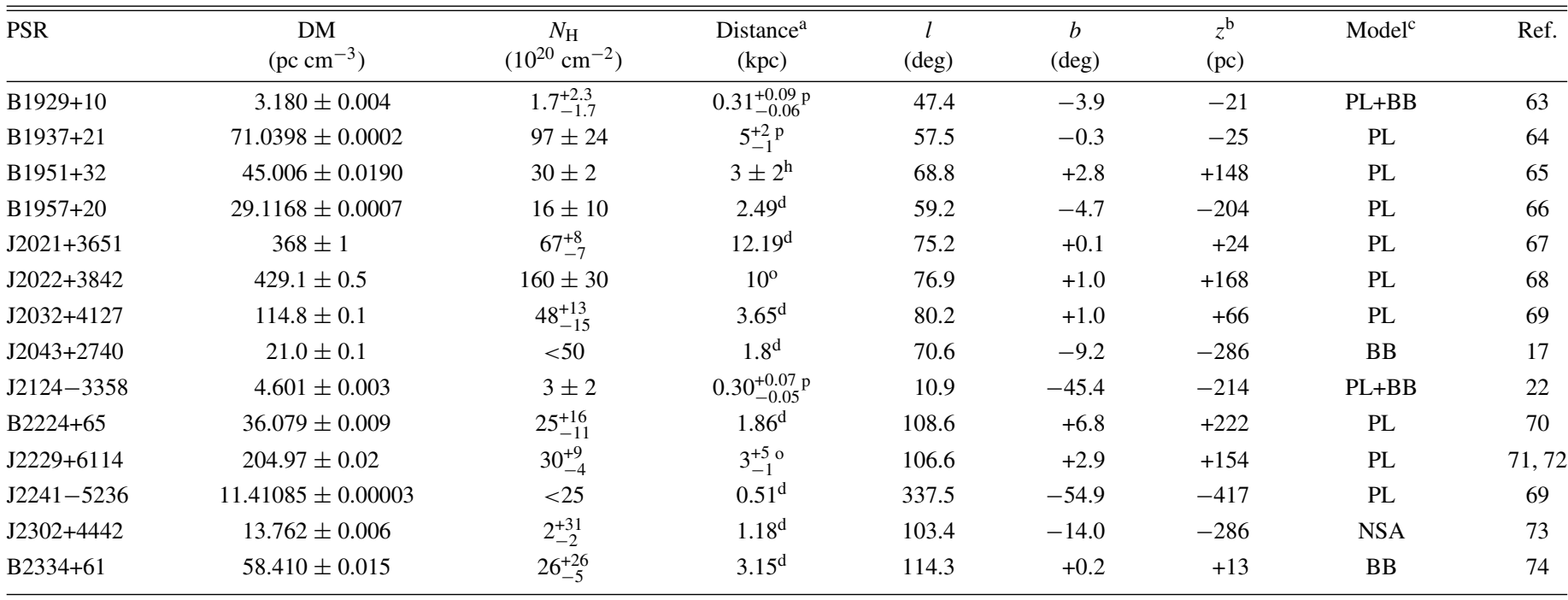

Notes. Uncertainties and upper limits on $N_{\mathrm{H}}$ are all scaled to the $90 \%$ confidence level, i.e., $1.6 \sigma$.

${ }^{a}$ Distance estimates are either parallax or H I absorption measurements adopted from Verbiest et al. (2012) and Deller et al. (2012) or from the DM using the NE2001 model (Cordes \& Lazio 2002). They are denoted by the letters p, h, and d, respectively. We did not attempt to quantify the uncertainties in the DM distances. The designation "o" indicates distance estimates based on other arguments; see the references for details.

${ }^{\mathrm{b}}$ Vertical distance from the Galactic plane calculated using the source distance and Galactic latitude $b$.

${ }^{\mathrm{c}}$ Spectral models used to obtain $N_{\mathrm{H}}$ : blackbody (BB), power law (PL), and neutron-star atmosphere (NSA). $N_{\mathrm{H}}$ values determined from the associated SNRs or PWNe are noted. The SNR spectra were fitted with Raymond-Smith (RS) and non-equilibrium ionization (VNEI) models.

d Schöck et al. (2010) reported $N_{\mathrm{H}}=1.15 \times 10^{21} \mathrm{~cm}^{-2}$, but mentioned that it is consistent with the previous result from Gaensler et al. (2002), which gave $N_{\mathrm{H}}=9.5 \times 10^{21} \mathrm{~cm}^{-2}$. Therefore, the former is assumed to be a typographical error and we adopt the value $1.15 \times 10^{22} \mathrm{~cm}^{-2}$.

References. (1) Bogdanov \& Grindlay 2009; (2) Posselt et al. 2012a; (3) Maselli et al. 2011; (4) Gotthelf et al. 2007; (5) Roberts et al. 1993; (6) Webb et al. 2004; (7) Tepedelenliğlu \& Ögelman 2007; (8) Durant et al. 2012; (9) Weisskopf et al. 2011; (10) Trimble 1973; (11) Ng et al. 2007; (12) Park et al. 2010; (13) Freedman et al. 2001; (14) Becker et al. 2005; (15) De Luca et al. 2005; (16) Possenti et al. 2008; (17) Becker et al. 2004; (18) LaMassa et al. 2008; (19) Zavlin \& Pavlov 2004; (20) Camilo et al. 2004; (21) Bogdanov et al. 2011a; (22) Zavlin 2006; (23) Gonzalez et al. 2006; (24) Ng et al. 2012; (25) Caswell et al. 2004; (26) Hughes et al. 2003; (27) Ransom et al. 2011; (28) Pavlov et al. 2011; (29) Negueruela et al. 2011; (30) Chang et al. 2012; (31) Renaud et al. 2010; (32) Ng et al. 2005; (33) Posselt et al. 2012b; (34) Kargaltsev et al. 2008; (35) Schöck et al. 2010; (36) Ng et al. 2011; (37) Pancrazi et al. 2012; (38) Kargaltsev et al. 2009; (39) Anderson et al. 2012; (40) Romani et al. 2005; (41) Zhu et al. 2011; (42) Hinton et al. 2007; (43) Olausen et al. 2013; (44) Bogdanov et al. 2010; (45) Reid \& Gizis 1998; (46) Kargaltsev et al. 2012; (47) Romani et al. 2010; (48) Holler et al. 2012; (49) Gaensler et al. 2004; (50) Gaensler et al. 2004; (51) Kaspi et al. 2001; (52) Kargaltsev et al. 2007; (53) Kargaltsev \& Pavlov 2007; (54) Bernardini et al. 2009; (55) Rea et al. 2009; (56) Bogdanov et al. 2011b; (57) Harris 1996; (58) Pavlov et al. 2008; (59) Matheson \& Safi-Harb 2010; (60) Shelton et al. 2004; (61) Claussen et al. 1996; (62) Temim et al. 2010; (63) Misanovic et al. 2008; (64) C.-Y. Ng et al. 2013, in preparation; (65) Li et al. 2005; (66) Guillemot et al. 2012; (67) Van Etten et al. 2008; (68) Arzoumanian et al. 2011; (69) Marelli 2012; (70) Hui et al. 2012; (71) Marelli et al. 2011; (72) Halpern et al. 2001; (73) Cognard et al. 2011; (74) McGowan et al. 2006.

taken from the ATNF Pulsar Catalog and distance estimates are obtained from parallax measurements, H I absorption measurements of the pulsars or the associated SNRs, or DM using the NE2001 Galactic electron density model (Cordes \& Lazio 2002). If available, parallax distances are always preferred since they are the most accurate. All parallax and $\mathrm{H}$ I distances are adopted from Verbiest et al. (2012) and references therein, and have been corrected for the Lutz-Kelker bias, except for PSR $\mathrm{J} 1023+0038$, which has a recent parallax measurement by Deller et al. (2012). For DM distances, we did not attempt to derive the uncertainties, but note that the fractional uncertainties could be $25 \%$ or larger (see, e.g., Camilo et al. 2009). Finally, there are exceptional cases in which previous studies argue for different distances than the DM-estimated ones. They are noted in the table. The pulsar $N_{\mathrm{H}}$ and DM are plotted against distance in Figures 2 and 3, respectively.

\section{ANALYSIS AND RESULTS}

Figure 1 shows a positive correlation between the pulsar DM and $N_{\mathrm{H}}$ values, with deviations ranging from a factor of a few to an order of magnitude. There are some obvious outliers, including the Vela pulsar (PSR B0833-45), the double pulsar (PSR J0737-3039), and PSR J1747-2809 in the Galactic center direction. To quantify the $\mathrm{DM}-N_{\mathrm{H}}$ correlation, we ignored pulsars with $N_{\mathrm{H}}$ upper limits and obtained a Pearson's correlation coefficient of 0.72. This is significant since the one-tailed probability of such a correlation arising by chance from unrelated variables is only $4 \times 10^{-5}$. More useful is an empirical relation between these two observables. We performed a linear fit to the data by minimizing the $\chi^{2}$ value. $N_{\mathrm{H}}$ measurements with fractional uncertainties larger than $80 \%$ or upper limits only (gray points in Figure 1) are excluded in the fit. We also ignored the Vela pulsar, which is located in the Gum Nebula inside the hot and low-density Local Bubble, and PSR B0540-69, which is in the Large Magellanic Cloud (LMC), because they seem unlikely to follow the DM- $N_{\mathrm{H}}$ correlation as would other Galactic sources. Only statistical uncertainties in $N_{\mathrm{H}}$ are considered in the $\chi^{2}$-fit since uncertainties in DM are negligible. Also, we did not attempt to model the systematic uncertainties, but we note that the ones introduced by different photoelectric absorption models and elemental abundances, or by cross-calibration between telescopes are only at a few percent level (see Wilms et al. 2000; Tsujimoto et al. 2011), relatively small compared to 

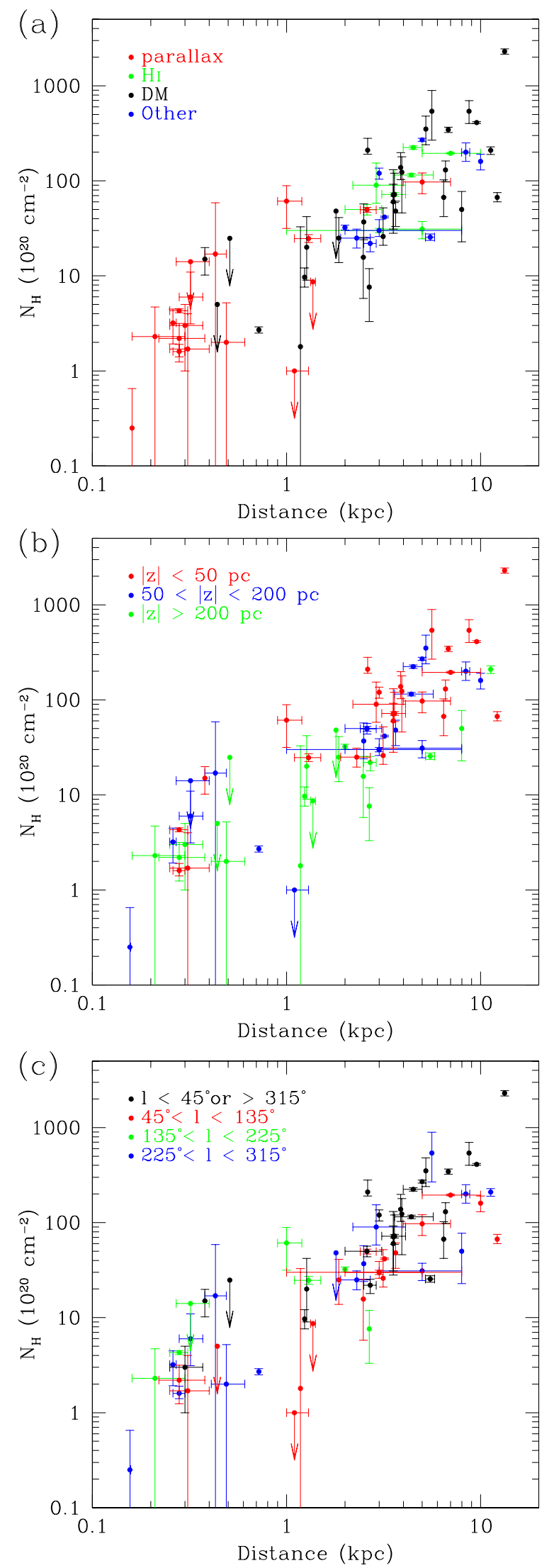

Figure 2. $N_{\mathrm{H}}$ vs. distance for Galactic pulsars. PSR B0540-69 is not shown here. (a) The colors indicate different types of distance measurement. We did not estimate the uncertainties for the DM distances. The same plot with different color schemes is shown in (b) and (c), indicating the pulsar vertical distance from the Galactic plane and their Galactic longitudes, respectively.

(A color version of this figure is available in the online journal.)
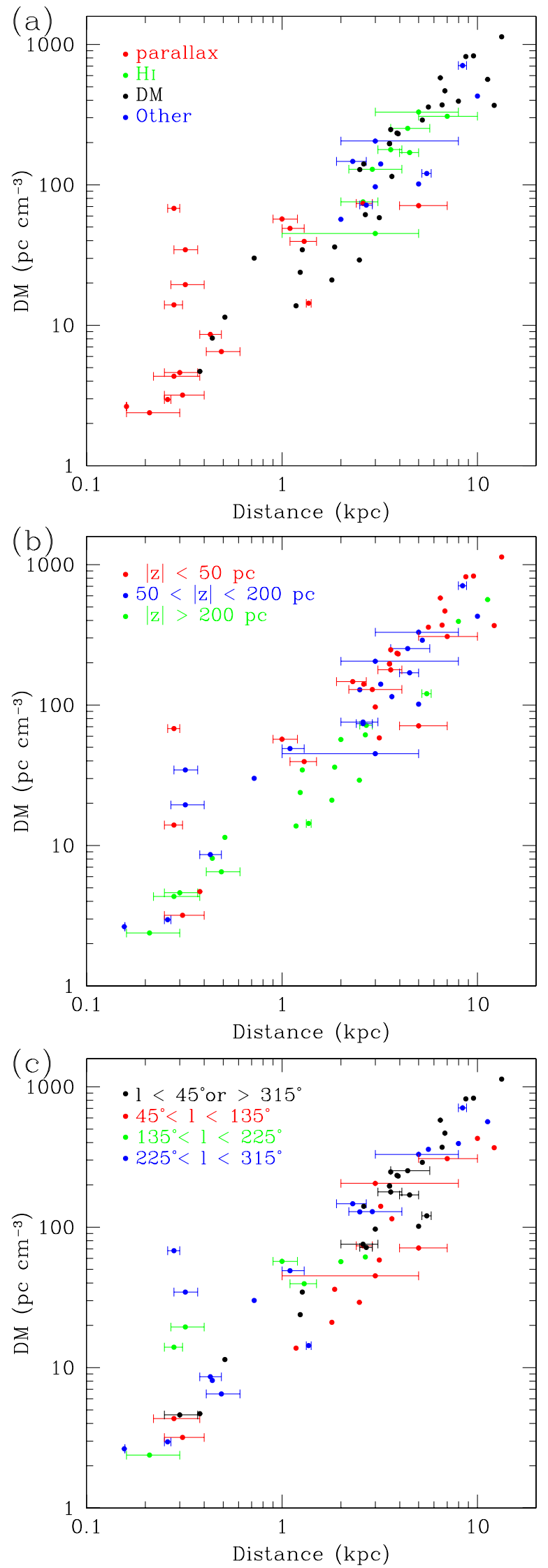

Figure 3. Same as Figure 2, but for DM vs. distance. Uncertainties in DM are not plotted, as they are smaller than the data points.

(A color version of this figure is available in the online journal.) 
the statistical uncertainties. Assuming $N_{\mathrm{H}}$ and DM are directly proportional, the best fit gives

$$
N_{\mathrm{H}}\left(10^{20} \mathrm{~cm}^{-2}\right)=0.30_{-0.09}^{+0.13} \mathrm{DM}\left(\mathrm{pc} \mathrm{cm}^{-3}\right),
$$

corresponding to an average ionization of $10_{-3}^{+4} \%$. The $90 \%$ confidence interval is quoted here, which is obtained from 10,000 simulations via bootstrapping resampling (Efron \& Tibshirani 1993). The result is plotted in Figure 1. We also tried fitting a more general linear relation by fitting the $y$-intercept as well, but found that the latter is consistent with zero at $90 \%$ confidence. If we ignore the measurement uncertainties in $N_{\mathrm{H}}$ and perform a least-squares fit, we obtain $N_{\mathrm{H}}\left(10^{20} \mathrm{~cm}^{-2}\right)=$ $0.83 \mathrm{DM}\left(\mathrm{pc} \mathrm{cm}^{-3}\right)$, giving a lower average ionization of $4 \%$.

To check if the DM $-N_{\mathrm{H}}$ relation could depend on the source location in the Galaxy, we divided the sample into groups according to their vertical height from the plane and their Galactic longitudes. The results are shown in Figures 1(b) and (c), respectively. In the high-DM regime, sources toward the Galactic center direction, e.g., PSRs J1747-2958 and J1747-2809, show a hint of a larger $N_{\mathrm{H}^{-}}$-to-DM ratio. However, the systematic variation is less clear at lower DM and our limited sample precludes a detailed analysis. In Figure 2, we plotted $N_{\mathrm{H}}$ against distance. This indicates a general correlation, albeit with a large scatter. There is also a hint that for sources at a similar distance, $N_{\mathrm{H}}$ is systematically larger near the Galactic plane (Figure 2(b)), however, the dependency on Galactic longitude is less clear (Figure 2(c)). The DM variation with distance is presented in Figure 3. While this may seem to exhibit a good correlation at large distances, we note that sources with DMderived distances provide no new information, only the NE2001 model prediction. In addition, there is a very large range of DMs for nearby pulsars around $300 \mathrm{pc}$, from $2.4 \pm 0.2 \mathrm{pc} \mathrm{cm}^{-3}$ for PSR J0108-1431 to $68 \pm 1.6 \mathrm{pc} \mathrm{cm}^{-3}$ for the Vela pulsar, spanning nearly a factor of 30 . Similar to $N_{\mathrm{H}}$, Figure $3(\mathrm{~b})$ also indicates a higher DM toward the Galactic plane.

\section{DISCUSSION}

We have investigated the $\mathrm{DM}-N_{\mathrm{H}}$ connection for 68 radio pulsars detected with Chandra or XMM-Newton. We found a good correlation between these two column densities, suggesting that free electrons in the Galaxy generally trace the interstellar gas. That said, some $N_{\mathrm{H}}$ values in Figure 1 show significant deviation from the best-fit line, by a factor of a few up to an order of magnitude. This could be attributed to inhomogeneity of the ISM, possibly due to molecular clouds, SNRs, or H II regions in the line of sight. Such an effect is more prominent for nearby sources, since the distribution of free electrons and interstellar gas is highly anisotropic around the Local Bubble (see Taylor \& Cordes 1993; Lallement et al. 2003). In particular, there is significant DM contribution from the Gum Nebula (Taylor \& Cordes 1993), resulting in a wide range of DMs for pulsars within $\sim 300 \mathrm{pc}$ (e.g., the Vela pulsar and PSR J0737-3039; see Figure 3). At large distances, local fluctuations are expected to average out and the scatter of $N_{\mathrm{H}}$ and DM with respect to distance likely arises from Galactic structure, such as the disk, spiral arms, and different scale heights of various ISM components (see Cox 2005). We have attempted to identify any systematic trends in DM and $N_{\mathrm{H}}$ with respect to source location. While Figures 2(b) and 3(b) hint at higher $N_{\mathrm{H}}$ and DM toward the Galactic plane, more sources are needed for a quantitative comparison with the detailed Galactic structure. Beyond our Galaxy, we note that while PSR B0540-69 in the
LMC was not used in the fit, its DM-to- $N_{\mathrm{H}}$ ratio lies close to the best-fit line in Figure 1. This is somewhat surprising because of the different interstellar abundances in the LMC than in our Galaxy (Russell \& Dopita 1992). We argue that this could merely be a coincidence rather than the general case. Indeed, the LMC contributes $90 \%$ of the $N_{\mathrm{H}}$ toward PSR B0540-69 (Park et al. 2010) but only two-thirds of the DM (Manchester et al. 2006).

The DM- $N_{\mathrm{H}}$ correlation can be used to estimate one quantity from the other, offering a useful tool for pulsar observations. For instance, radio pulsations have been claimed from the magnetar 4U 0142+61 with a DM of $27 \pm 5 \mathrm{pc} \mathrm{cm}^{-3}$ (Malofeev et al. 2010). Given its $N_{\mathrm{H}}$ value of $9.6 \pm 0.2 \times 10^{21} \mathrm{~cm}^{-2}$ (Göhler et al. 2005), the claimed DM seems somewhat small when compared to other sources of similar $N_{\mathrm{H}}$ in Figure 1. For X-ray observations, there are many cases requiring a priori knowledge of $N_{\mathrm{H}}$, including flux estimates when planning for new observations, measuring the intrinsic spectra of faint sources, and deriving luminosity limits for non-detection. In many previous studies, $N_{\mathrm{H}}$ is inferred from the DM by assuming one free electron per 10 neutral hydrogen atoms (e.g., Kargaltsev et al. 2007; Camilo et al. 2012). Our result directly confirms that this is a reasonable approximation, but as a caveat, the scatter in $N_{\mathrm{H}}$ is typically a factor of a few up to an order of magnitude.

In addition to DM, the total Galactic $\mathrm{H}$ i column density from $21 \mathrm{~cm}$ radio surveys (e.g., Kalberla et al. 2005) and $A_{\mathrm{V}}$ have also been used as proxies for the X-ray absorption (e.g., Olausen et al. 2013). These $N_{\mathrm{H}}$ estimates are plotted in Figure 4. It is clear that some X-ray-inferred $N_{\mathrm{H}}$ values exceed the total H i column density of the Galaxy. As shown in the figure, the latter saturates at $\sim 10^{22} \mathrm{~cm}^{-2}$, resulting in gross underestimates for highDM $\left(\gtrsim 100 \mathrm{pc} \mathrm{cm}^{-3}\right)$ or distant $(\gtrsim 3 \mathrm{kpc})$ pulsars. It has been reported that at high Galactic column densities $\gtrsim 10^{21} \mathrm{~cm}^{-2}$, which occur at low Galactic latitudes, the X-ray absorption columns are generally larger than the H I columns by a factor of 1.5-3 (Arabadjis \& Bregman 1999; Baumgartner \& Mushotzky 2006). This agrees with our result and indicates significant X-ray absorption due to molecular clouds rather than neutral hydrogen atoms (see Willingale et al. 2013), hence, the H i column may not be a good tracer for the X-ray absorption.

$A_{\mathrm{V}}$, on the other hand, is caused by grains of the same heavy elements that give rise to X-ray absorption, and therefore, it highly correlates with $N_{\mathrm{H}}$ (e.g., Predehl \& Schmitt 1995; Güver $\&$ Ozel 2009). Given a pulsar's position and distance, $A_{V}$ can be estimated from the three-dimensional extinction maps of the Galaxy (e.g., Drimmel et al. 2003), and then $N_{\mathrm{H}}$ can be deduced from the empirical relation $N_{\mathrm{H}}\left(\mathrm{cm}^{-2}\right)=2.21 \times 10^{21} A_{\mathrm{V}}(\mathrm{mag})$ (Güver \& Özel 2009). As shown in Figure 4, this method seems to give the best agreement between measured and predicted values, especially for the highest $N_{\mathrm{H}}$ pulsars. It is worth noting that in some cases DMs were used to infer the pulsar distances, which then give $A_{\mathrm{V}}$ and $N_{\mathrm{H}}$. This generally provides better results than directly employing the $\mathrm{DM}-N_{\mathrm{H}}$ correlation. We believe that this is because the $A_{\mathrm{V}}$ map reflects the distribution of heavy elements in the Galaxy, whereas this crucial information cannot be obtained from DM.

\section{CONCLUSION AND OUTLOOK}

We have compiled a list of 68 pulsar $N_{\mathrm{H}}$ measurements reported in the literature using Chandra and XMM-Newton observations, and compared the $N_{\mathrm{H}}$ values with the DMs and distances. Our results show a good correlation between DM and $N_{\mathrm{H}}$, with a correlation coefficient of 0.72 . We obtained an 

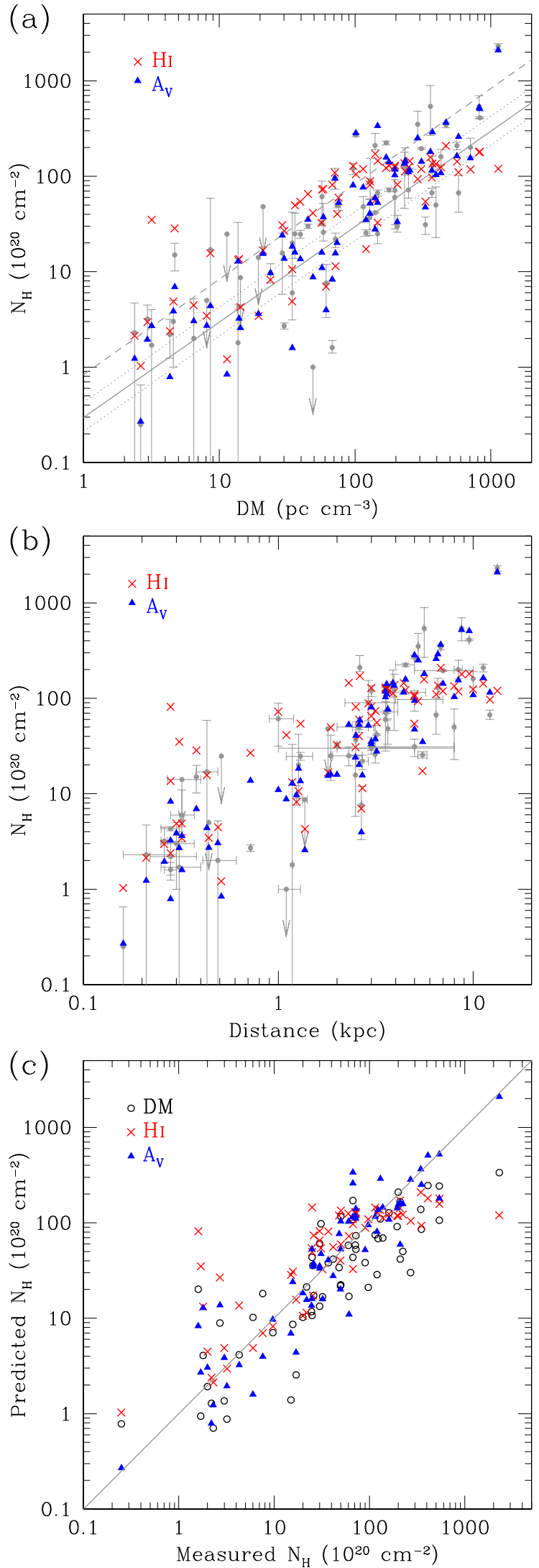

Figure 4. Comparison between $N_{\mathrm{H}}$ estimates and measurements. The underlying plots in (a) and (b) are the same as Figures 1 and 2, respectively. The red crosses indicate the total line-of-sight Galactic H i column densities for each pulsar, given by $21 \mathrm{~cm}$ radio observations (Kalberla et al. 2005). The blue triangles show estimates based on $A_{\mathrm{V}}$ (see text). In (c), the open circles represent predictions from our best-fit $\mathrm{DM}-\mathrm{N}_{\mathrm{H}}$ relation.

(A color version of this figure is available in the online journal.) empirical linear relation $N_{\mathrm{H}}\left(10^{20} \mathrm{~cm}^{-2}\right)=0.30_{-0.09}^{+0.13} \mathrm{DM}$ $\left(\mathrm{pc} \mathrm{cm}^{-3}\right)$, implying an average ionization of $10_{-3}^{+4} \%$. This confirms the ratio of one free electron to 10 neutral hydrogen atoms commonly used in previous studies. Our finding provides a useful tool to estimate $N_{\mathrm{H}}$ from DM. We compare it to other $N_{\mathrm{H}}$ estimates based on the neutral hydrogen column density and $A_{\mathrm{V}}$, and find that the latter gives the best results, while $\mathrm{H}$ I and our empirical DM- $N_{\mathrm{H}}$ relation tend to give underestimates in the high- $N_{\mathrm{H}}$ regime.

The next generation of X-ray missions, including eROSITA (Predehl et al. 2010) and the proposed Neutron Star Interior Composition Explorer (Gendreau et al. 2012), will significantly expand the pulsar $N_{\mathrm{H}}$ sample. In addition, the foreseen Square Kilometer Array can provide parallax measurements of a few thousand radio pulsars (Smits et al. 2011). Together these will allow a detailed study of the $\mathrm{DM}-N_{\mathrm{H}}$ relation in different parts of the Galaxy and its connection with the Galactic structure. In addition to pulsars, it should be possible to compile a database of $N_{\mathrm{H}}$ measurements for other Galactic X-ray sources, such as stars, SNRs, cataclysmic variables, stellar clusters, white dwarfs, and X-ray binaries, and compare with their distances to build a three-dimensional $N_{\mathrm{H}}$ map of our Galaxy.

We thank Oleg Kargaltsev and Slavko Bogdanov for suggesting a list of X-ray-emitting radio pulsars, and Anne Archibald, Antoine Bouchard, and Ryan Lynch for discussion. We acknowledge the anonymous referee for useful suggestions. V.M.K. holds the Lorne Trottier Chair in Astrophysics and Cosmology and a Canadian Research Chair in Observational Astrophysics. This work was supported by NSERC via a Discovery Grant, by FQRNT via the Centre de Recherche Astrophysique du Québec, by CIFAR, and a Killam Research Fellowship.

\section{REFERENCES}

Anderson, G. E., Gaensler, B. M., Slane, P. O., et al. 2012, ApJ, 751, 53 Arabadjis, J. S., \& Bregman, J. N. 1999, ApJ, 510, 806

Arzoumanian, Z., Gotthelf, E. V., Ransom, S. M., et al. 2011, ApJ, 739, 39

Baumgartner, W. H., \& Mushotzky, R. F. 2006, ApJ, 639, 929

Becker, W., \& Aschenbach, B. 2002, in Neutron Stars, Pulsars, and Supernova Remnants, ed. W. Becker, H. Lesch, \& J. Trümper (Garching: Max-PlanckInstitut für Extraterrestrische Physik), 64

Becker, W., Jessner, A., Kramer, M., Testa, V., \& Howaldt, C. 2005, ApJ, 633, 367

Becker, W., Weisskopf, M. C., Tennant, A. F., et al. 2004, ApJ, 615, 908

Bernardini, F., Israel, G. L., Dall'Osso, S., et al. 2009, A\&A, 498, 195

Bogdanov, S., Archibald, A. M., Hessels, J. W. T., et al. 2011a, ApJ, 742, 97

Bogdanov, S., \& Grindlay, J. E. 2009, ApJ, 703, 1557

Bogdanov, S., van den Berg, M., Heinke, C. O., et al. 2010, ApJ, 709, 241

Bogdanov, S., van den Berg, M., Servillat, M., et al. 2011b, ApJ, 730, 81

Camilo, F., Gaensler, B. M., Gotthelf, E. V., Halpern, J. P., \& Manchester, R. N. 2004, ApJ, 616, 1118

Camilo, F., Ng, C.-Y., Gaensler, B. M., et al. 2009, ApJL, 703, L55

Camilo, F., Ransom, S. M., Chatterjee, S., Johnston, S., \& Demorest, P. 2012, ApJ, 746, 63

Camilo, F., Ransom, S. M., Halpern, J. P., et al. 2006, Natur, 442, 892

Camilo, F., Ransom, S. M., Halpern, J. P., \& Reynolds, J. 2007, ApJL, 666, L93

Caswell, J. L., McClure-Griffiths, N. M., \& Cheung, M. C. M. 2004, MNRAS, 352, 1405

Chang, C., Pavlov, G. G., Kargaltsev, O., \& Shibanov, Y. A. 2012, ApJ, 744, 81

Claussen, M. J., Wilking, B. A., Benson, P. J., et al. 1996, ApJS, 106, 111

Cognard, I., Guillemot, L., Johnson, T. J., et al. 2011, ApJ, 732, 47

Cordes, J. M., \& Lazio, T. J. W. 2002, arXiv:astro-ph/0207156

Cox, D. P. 2005, ARA\&A, 43, 337

De Luca, A., Caraveo, P. A., Mereghetti, S., Negroni, M., \& Bignami, G. F. 2005, ApJ, 623, 1051

Deller, A. T., Archibald, A. M., Brisken, W. F., et al. 2012, ApJL, 756, L25 
Drimmel, R., Cabrera-Lavers, A., \& López-Corredoira, M. 2003, A\&A, 409, 205

Durant, M., Kargaltsev, O., Pavlov, G. G., et al. 2012, ApJ, 746, 6

Efron, B., \& Tibshirani, R. J. 1993, An Introduction to the Bootstrap (New York: Chapman \& Hall)

Freedman, W. L., Madore, B. F., Gibson, B. K., et al. 2001, ApJ, 553, 47

Gaensler, B. M., Arons, J., Kaspi, V. M., et al. 2002, ApJ, 569, 878

Gaensler, B. M., van der Swaluw, E., Camilo, F., et al. 2004, ApJ, 616, 383

Gendreau, K. C., Arzoumanian, Z., \& Okajima, T. 2012, Proc. SPIE, 8443, 13

Gil, J., Haberl, F., Melikidze, G., et al. 2008, ApJ, 686, 497

Göhler, E., Wilms, J., \& Staubert, R. 2005, A\&A, 433, 1079

Gonzalez, M. E., Kaspi, V. M., Pivovaroff, M. J., \& Gaensler, B. M. 2006, ApJ, 652,569

Gotthelf, E. V., Helfand, D. J., \& Newburgh, L. 2007, ApJ, 654, 267

Guillemot, L., Johnson, T. J., Venter, C., et al. 2012, ApJ, 744, 33

Güver, T., \& Özel, F. 2009, MNRAS, 400, 2050

Halpern, J. P., Gotthelf, E. V., Leighly, K. M., \& Helfand, D. J. 2001, ApJ, 547,323

Harris, W. E. 1996, AJ, 112, 1487

Hinton, J. A., Funk, S., Carrigan, S., et al. 2007, A\&A, 476, L25

Holler, M., Schöck, F. M., Eger, P., et al. 2012, A\&A, 539, A24

Hughes, J. P., Slane, P. O., Park, S., Roming, P. W. A., \& Burrows, D. N. 2003, ApJL, 591, L139

Hui, C. Y., Huang, R. H. H., Trepl, L., et al. 2012, ApJ, 747, 74

Kalberla, P. M. W., Burton, W. B., Hartmann, D., et al. 2005, A\&A, 440, 775

Kargaltsev, O., Durant, M., Misanovic, Z., \& Pavlov, G. G. 2012, Sci, 337, 946

Kargaltsev, O., Misanovic, Z., Pavlov, G. G., Wong, J. A., \& Garmire, G. P. 2008, ApJ, 684, 542

Kargaltsev, O., \& Pavlov, G. G. 2007, ApJ, 670, 655

Kargaltsev, O., \& Pavlov, G. G. 2008, in AIP Conf. Proc. 983, 40 Years of Pulsars: Millisecond Pulsars, Magnetars and More, ed. C. Bassa, Z. Wang, A. Cumming, \& V. M. Kaspi (Melville, NY: AIP), 171

Kargaltsev, O., \& Pavlov, G. G. 2010, in AIP Conf. Proc. 1248, X-ray Astronomy 2009; Present Status, Multi-Wavelength Approach and Future Perspectives, ed. A. Comastri, L. Angelini, \& M. Cappi (Melville, NY: AIP), 25

Kargaltsev, O., Pavlov, G. G., \& Garmire, G. P. 2007, ApJ, 660, 1413

Kargaltsev, O., Pavlov, G. G., \& Wong, J. A. 2009, ApJ, 690, 891

Kaspi, V. M., Gotthelf, E. V., Gaensler, B. M., \& Lyutikov, M. 2001, ApJL, 562, L163

Lallement, R., Welsh, B. Y., Vergely, J. L., Crifo, F., \& Sfeir, D. 2003, A\&A, 411,447

LaMassa, S. M., Slane, P. O., \& de Jager, O. C. 2008, ApJL, 689, L121

Levin, L., Bailes, M., Bates, S., et al. 2010, ApJL, 721, L33

Li, X. H., Lu, F. J., \& Li, T. P. 2005, ApJ, 628, 931

Malofeev, V. M., Teplykh, D. A., \& Malov, O. I. 2010, ARep, 54, 995

Manchester, R. N., Fan, G., Lyne, A. G., Kaspi, V. M., \& Crawford, F. 2006, ApJ, 649, 235

Manchester, R. N., Hobbs, G. B., Teoh, A., \& Hobbs, M. 2005, AJ, 129, 1993

Marelli, M. 2012, PhD thesis, Univ. Insubria

Marelli, M., De Luca, A., \& Caraveo, P. A. 2011, ApJ, 733, 82

Maselli, A., Cusumano, G., Massaro, E., et al. 2011, A\&A, 531, A153

Matheson, H., \& Safi-Harb, S. 2010, ApJ, 724, 572

McGowan, K. E., Zane, S., Cropper, M., Vestrand, W. T., \& Ho, C. 2006, ApJ, 639, 377

Misanovic, Z., Pavlov, G. G., \& Garmire, G. P. 2008, ApJ, 685, 1129
Negueruela, I., Ribó, M., Herrero, A., et al. 2011, ApJL, 732, L11

Ng, C.-Y., Kaspi, V. M., Dib, R., et al. 2011, ApJ, 729, 131

Ng, C.-Y., Kaspi, V. M., Ho, W. C. G., et al. 2012, ApJ, 761, 65

Ng, C.-Y., Roberts, M. S. E., \& Romani, R. W. 2005, ApJ, 627, 904

Ng, C.-Y., Romani, R. W., Brisken, W. F., Chatterjee, S., \& Kramer, M. 2007, ApJ, 654, 487

Olausen, S. A., Zhu, W. W., Vogel, J. K., et al. 2013, ApJ, 764, 1

Pancrazi, B., Webb, N. A., Becker, W., et al. 2012, A\&A, 544, A108

Park, S., Hughes, J. P., Slane, P. O., Mori, K., \& Burrows, D. N. 2010, ApJ, 710, 948

Pavlov, G. G., Chang, C., \& Kargaltsev, O. 2011, ApJ, 730, 2

Pavlov, G. G., Kargaltsev, O., \& Brisken, W. F. 2008, ApJ, 675, 683

Pavlov, G. G., Kargaltsev, O., Garmire, G. P., \& Wolszczan, A. 2007, ApJ, 664, 1072

Posselt, B., Arumugasamy, P., Pavlov, G. G., et al. 2012a, ApJ, 761, 117

Posselt, B., Pavlov, G. G., Manchester, R. N., Kargaltsev, O., \& Garmire, G. P. 2012b, ApJ, 749, 146

Possenti, A., Cerutti, R., Colpi, M., \& Mereghetti, S. 2002, A\&A, 387, 993

Possenti, A., Rea, N., McLaughlin, M. A., et al. 2008, ApJ, 680, 654

Predehl, P., Andritschke, R., Böhringer, H., et al. 2010, Proc. SPIE, 7732, 23

Predehl, P., \& Schmitt, J. H. M. M. 1995, A\&A, 293, 889

Ransom, S. M., Ray, P. S., Camilo, F., et al. 2011, ApJL, 727, L16

Rea, N., McLaughlin, M. A., Gaensler, B. M., et al. 2009, ApJL, 703, L41

Reid, I. N., \& Gizis, J. E. 1998, AJ, 116, 2929

Renaud, M., Marandon, V., Gotthelf, E. V., et al. 2010, ApJ, 716, 663

Roberts, D. A., Goss, W. M., Kalberla, P. M. W., Herbstmeier, U., \& Schwarz, U. J. 1993, A\&A, 274, 427

Romani, R. W., Ng, C.-Y., Dodson, R., \& Brisken, W. 2005, ApJ, 631, 480

Romani, R. W., Shaw, M. S., Camilo, F., Cotter, G., \& Sivakoff, G. R. 2010, ApJ, 724, 908

Russell, S. C., \& Dopita, M. A. 1992, ApJ, 384, 508

Schöck, F. M., Büsching, I., de Jager, O. C., Eger, P., \& Vorster, M. J. 2010, A\&A, 515, A109

Seward, F. D., \& Wang, Z.-R. 1988, ApJ, 332, 199

Shelton, R. L., Kuntz, K. D., \& Petre, R. 2004, ApJ, 611, 906

Smits, R., Tingay, S. J., Wex, N., Kramer, M., \& Stappers, B. 2011, A\&A, 528, A108

Taylor, J. H., \& Cordes, J. M. 1993, ApJ, 411, 674

Temim, T., Slane, P., Reynolds, S. P., Raymond, J. C., \& Borkowski, K. J. 2010, ApJ, 710, 309

Tepedelenliğlu, E., \& Ögelman, H. 2007, ApJ, 658, 1183

Trimble, V. 1973, PASP, 85, 579

Tsujimoto, M., Guainazzi, M., Plucinsky, P. P., et al. 2011, A\&A, 525, A25

Van Etten, A., Romani, R. W., \& Ng, C.-Y. 2008, ApJ, 680, 1417

Verbiest, J. P. W., Weisberg, J. M., Chael, A. A., Lee, K. J., \& Lorimer, D. R. 2012, ApJ, 755, 39

Webb, N. A., Olive, J.-F., \& Barret, D. 2004, A\&A, 417, 181

Weisskopf, M. C., Tennant, A. F., Yakovlev, D. G., et al. 2011, ApJ, 743, 139

Willingale, R., Starling, R. L. C., Beardmore, A. P., Tanvir, N. R., \& O’Brien, P. T. 2013, MNRAS, 431, 394

Wilms, J., Allen, A., \& McCray, R. 2000, ApJ, 542, 914

Zavlin, V. E. 2006, ApJ, 638, 951

Zavlin, V. E., \& Pavlov, G. G. 2004, ApJ, 616, 452

Zhu, W. W., Kaspi, V. M., McLaughlin, M. A., et al. 2011, ApJ, 734, 44 